\title{
Multiscale Feature-Preserving Smoothing of Tomographic Data
}

\author{
Nassim Jibai \\ INRIA, Grenoble University
}

\author{
Kartic Subr \\ University College London
}

\author{
Nicolas Holzschuch \\ INRIA, Grenoble University
}

\begin{abstract}
Computer tomography (CT) has wide application in medical imaging and reverse engineering. Due to the limited number of projections used in reconstructing the volume, the resulting 3D data is typically noisy. Contouring such data, for surface extraction, yields surfaces with localised artifacts of complex topology. To avoid such artifacts, we propose a method for feature-preserving smoothing of CT data. The smoothing is based on anisotropic diffusion, with a diffusion tensor designed to smooth noise up to a given scale, while preserving features. We compute these diffusion kernels from the directional histograms of gradients around each voxel, using a fast GPU implementation.
\end{abstract}

Keywords: Feature-preserving smoothing, anisotropic diffusion

\section{Introduction}

Computer tomography is an effective way of digitally scanning objects with complex and intricate geometry. Example applications include medical imaging and reverse-engineering of closed objects in a non-destructive acquisition process. Volumetric data from inverse tomography are prone to artifacts due to imperfectly acquired projections. These imperfections have multiple causes: high contrasts; lack of captor sensitivity; too few projections; nonmonochromaticity of the X-Ray source, imperfect stability of the $\mathrm{X}$-Ray source; pixel defects on the captor; non-uniformity of the absorbtion process accross wavelengths.

A popular approach to smooth such 3D data is to use geometric diffusion. Geometric diffusion smoothes orthogonal to the gradient in the volume and therefore preserves planar regions, while blurring edges and corners. More general anisotropy has also been proposed where the flatness of the diffusion kernel is adapted to the gradient of the volume [Schaap et al. 2008], or the structure tensor [Frangakis and Hegerl 2001]. In all these cases, the diffusion kernel is radially symmetric (two eigenvalues are equal).

We propose a new method of computing a diffusion tensor for each voxel based on the distribution of gradients in its neighborhood. We build a radially asymmetric diffusion tensor by combining the geometric diffusion tensors of the gradients in the neighborhood of each voxel. Two input parameters control the extent of smoothing: the size of the neighborhood and the number of diffusion steps. We propose an efficient method to compute these tensors on GPU.

\section{Multi-Scale feature-preserving diffusion}

We need our diffusion kernel to respect the constraints of the individual geometric-diffusion kernels for each gradient in the neighborhood of the current voxel. Thus, we use the geometric average of the geometric diffusion kernels. To avoid discretization artifacts, we compute the continuous directional histogram [Kass and Solomon 2010] $h_{x}$ of gradients $\nabla_{f}(x)$ of the volumetric data $f$ around each point $x$ in the volume using a Von Mises kernel $K$. The value of the histogram for a given direction is obtained by integrating over gradients of nearby voxels with a gaussian weight $g_{\alpha}$ of variance $\alpha$ :

$h_{x}(\omega)=\frac{1}{\mathcal{K}} \int_{V} K\left(\nabla_{f}(y), \omega\right) g_{\alpha}(x-y) d y=\frac{1}{\mathcal{K}} K\left(\nabla_{f}(),. \omega\right) \otimes g_{\alpha}$

where $\alpha$ is the input feature size, $\mathcal{K}$ is the normalizing constant that accounts for the gaussian $g_{\alpha}$ and the Vom Mises kernel $K$. Thus,

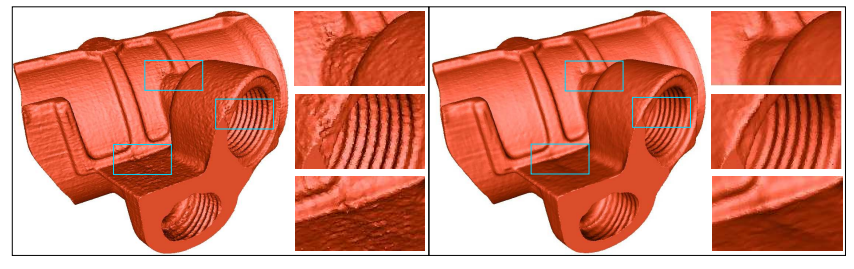

Figure 1: Left: A contour surface extracted from noisy tomographic data contains surface noise and several topological artifacts such as small handles and holes. Right: The surface extracted from our smoothed volume is clean, and yet small features, such as the thread in the screw, and sharp edges have been preserved.

we compute $h(\omega)$ for the entire volume at once along each histogram direction $\omega$ using only two 3D FFTs. For a direction $\omega$, the geometric diffusion kernel is a 3D gaussian $e^{-{ }^{t} x M_{g}(\omega) x}$ where $M_{g}(\omega)$ is a $3 \mathrm{D}$ symmetric matrix. To build a diffusion kernel that accounts for the entire histogram of directions around voxel $x$ we compute the geometric average of gaussian filters weighted by the histogram for each direction. The matrix $M_{x}$ of this kernel is therefore the integral of matrices $M_{g}$ for all directions weighted by the gradient histogram in this direction:

$$
M_{x}=\frac{1}{\mathcal{H}} \int_{\Omega} h_{x}(\omega) M_{g}(\omega) d \omega
$$

Because we compute $h(\omega)$ for all voxels at once, we can numerically compute $M_{x}$ for all voxels $x$ by sweeping accross a finite set of directions $\omega_{i}$. The diffusion then uses the three eigenvectors $v_{i}$ and eigenvalues $\lambda_{i}$ of $M_{x}$, and the Hessian $H$ of the volume:

$$
\frac{\partial v}{\partial t}=\sum_{i=1,2,3} \lambda_{i}{ }^{t} v_{i} H v_{i}
$$

The memory cost of computing the diffusion kernel per voxel is that of storing the symmetric matrix $M_{x}$ plus the normalizing constant $\mathcal{H}$, i.e. only 7 floats per voxel. We can therefore treat volumes up to $512^{3}$ voxels in $4 G b$ of memory. It is possible to compute the diffusion kernels block per block as well, with a proper overlap between the blocks. We used our method to improve tomographic data (See Fig. 1). Our method is fast, as we are able to compute kernels in a $256^{3}$ volume in 700 seconds for 642 directions using a $10^{3}$ feature size (while the same calculation needs hours on CPU). Each diffusion step then takes less than $5 \mathrm{sec}$. Our technique can be extended to repair surfaces as well, while eliminating artifacts of arbitrary topology up to a given scale, if by first converting the mesh into 3D level set data.

\section{References}

FRANGAKIS, A. S., AND HEgERL, R. 2001. Noise reduction in electron tomographic reconstructions using nonlinear anisotropic diffusion. Journal of Structural Biology $135,3,239-250$.

Kass, M., And Solomon, J. 2010. Smoothed local histogram filters. ACM Trans. Graph. 29 (July), 100:1-100:10.

SchaAp, M., Schilham, A., Zuiderveld, K., Prokop, M., Vonken, E.-J., AND NiESSEN, W. 2008. Fast noise reduction in computed tomography for improved 3-d visualization. Medical Imaging, IEEE Transactions on 27, 8, 1120 -1129 . 